\title{
Journal of Dairy Science Editorial Board and Ad Hoc Reviewers, 2009
}

The American Dairy Science Assoication and the Journal of Dairy Science gratefully acknowledge the following individuals who have served as members of the editorial board or as ad hoc reviewers in 2009.

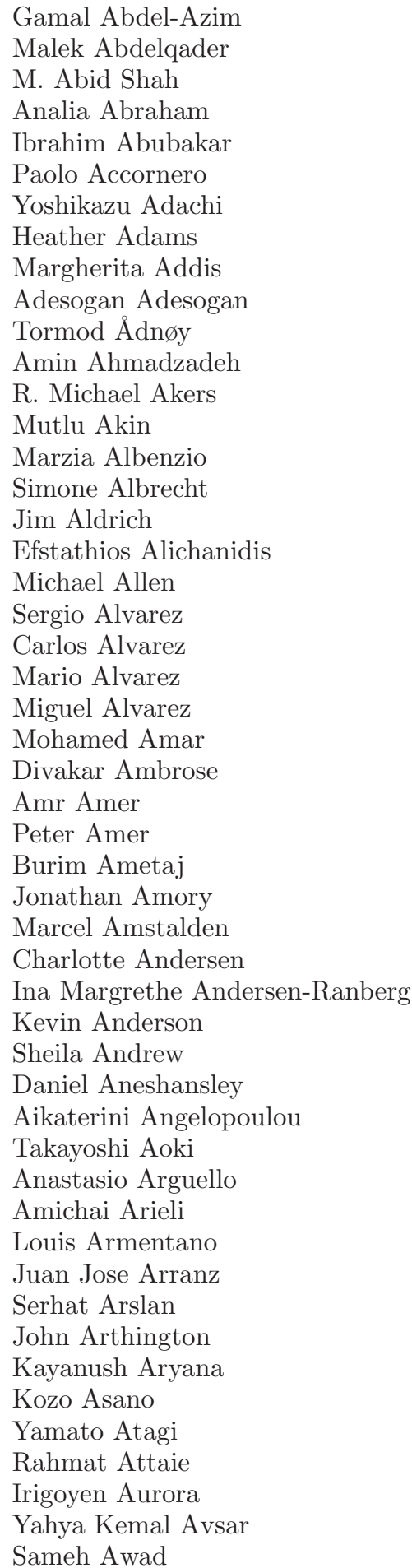

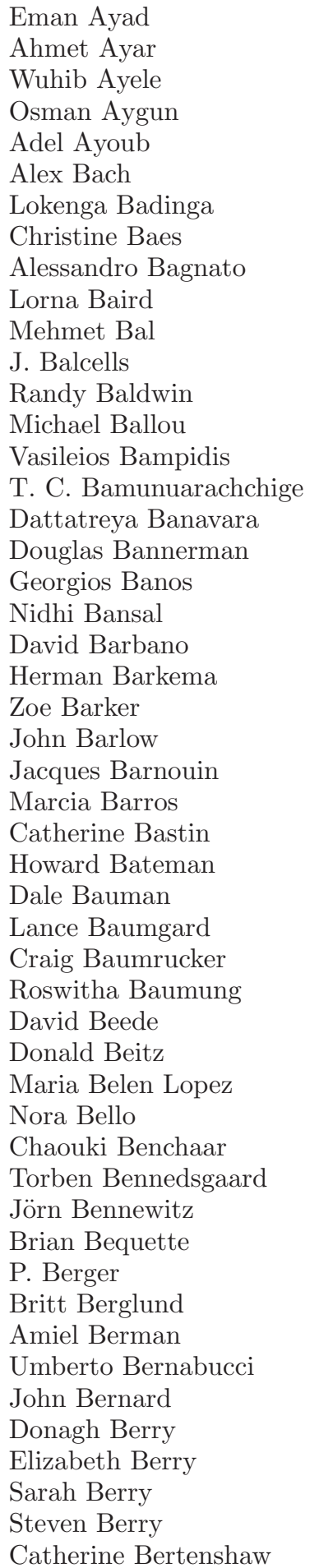

Eman Ayad

Osman Aygun

Adel Ayoub

Lokenga Badinga

Christine Baes

Alessandro Bagnato

Randy Baldwin

T. C. Bamunuarachchige

Georgios Banos

Nidhi Bansal

David Barbano

Herman Barkema

John Barlow

Jacques Barnouin

Marcia Barros

Dale Bauman

Lance Baumgard

Roswitha Baumung

David Beede

Donald Beitz

Maria Belen Lopez

ora Bello

Torben Bennedsgaard

witz

Umberto Bernabucci

John Bernard

Donagh Berry

Elizabeth Berry

Steven Berry

Catherine Bertenshaw
Sandra Bertics

Wagner Beskow

Greg Bethard

Wopke Beukema

Jeffrey Bewley

Rodrigo Bicalho

Stefano Biffani

Piter Bijma

Todd Bilby

Mario Binelli

Ana Binetti

J. Bishop

Stephen Bishop

Giovanni Bittante

Michael Black

Maiara Blagitz

Marta Blanch

Dale Blasi

Elliot Block

Patrick Blondin

Juerg Blum

Gerd Bobe

Guenther Boehm

Jamie Boehmer

Paul J. Boettcher

Jarmila Bohmanova

Didier Boichard

Yves Boisclair

Heinrich Bollwein

Adriana Bonanno

Kathryn Boor

Fernando Borderas

Sarah Boucher

Veronique Boulanger

Claire Bourlieu

H. Bovenhuis

Barry Bradford

Randall Brandsma

Martin Braunschweig

David Bravo

David Bray

James Breen

Gerhard Breves

Allen Bridges

Mercedes Brighenti

Guillaume Brisson

Andre Brito

Jack Britt

Michel Britten

Glen Broderick 
Arieh Brosh

Sue Brotherstone

Micheal Brouk

Rupert Bruckmaier

Keith Bryan

Mateo Budinich

Albert (Bart) Buitenhuis

Marisa Bunning

Christian Burvenich

Ron Butler

Stephen Butler

John Byatt

Andrea Cabiddu

Victor Cabrera

Margherita Caccamo

Roger Cady

Wei-Min Cai

Yimin Cai

Gerardo Caja

Alicia Califano

Douglas Call

Todd Callaway

Lien Callewaert

Sergio Calsamiglia

Mario Calus

J. H. Calvo

M. Angeles Calvo Torras

C Cambillau

Taissa Canaes

Christopher Canale

Fabiola Canavesi

Wenceslao Canet

Raul Cano

Rodolpho Canseco

John Cant

Rodolfo Cantet

Zhijun Cao

Nynne Capion

Jude Capper

Anthony Capuco

Vincent Cardot

Emma Carlén

Miguel Carneiro

Paolo Carnier

Anna Caroli

Guillermo Carrillo-Castaneda

Maria Dolores Carro

Antonello Carta

David Casper

Martino Cassandro

Bennet Cassell

Lorena Castillejos

Héctor Castillo-Juárez

Francesca Cavalieri

Philippe Cayot

Pietro Celi

Marcelo Chaffer

Nuria Chapinal
Jean-Michel Chardigny

Larry Chase

Ricardo Chebel

Prasanth Chelikani

Ming-Ju Chen

John Chenault

Jacques Chesnais

Stefania Chessa

Hugh Chester-Jones

Munashe Chigerwe

Yves Chilliard

Babu Chinnasamy

Johanne Chiquette

Jean-Marc Chobert

P. Yvan Chouinard

Mary Christman

Giorgos Christodoulopoulos

Chishih Chu

Sumru Citak

Mary Clapperton

Stephanie Clark

Wayne Coblentz

Michael Coffey

Tim M. Cogan

Patti Coggins

Wendie Cohick

John Cole

Robert Collier

Dario Colombatto

Bernard Conlin

Timothy Connelly

Erin Connor

Peter Constable

Antonio Contreras

Nigel Cook

Reinaldo Cooke

Tim Coolbear

F. J. H. Corichi

Benjamin Corl

Agnès Cornu

Milena Corredig

Georges Corrieu

Douglas Cosby

S. Cosentino

Elvira Costell

Sandra Costello

Gerardus Cramer

Mark Crowe

Christian Cruywagen

Beatriz Cuartas-Uribe

Kevin Cummings

Keith Cummins

John Currin

Wladimir da Silva

Hans Daetwyler

Geoff Dahl

Robert Dailey

Joseph Dalton
Kristy Daniels

Heather Dann

Ingrid David

Mike Davidson

Ross Davidson

Stephen Davis

Michael Day

Bernard De Baets

Nele de Belie

Yvette de Haas

Evert de Jong

Dirk-Jan De Koning

Miguel de la Fuente

Massimo De Marchi

E. C. P. De Martinis

Pasquale De Palo

Anne Marie de Passillé

Sander de Roos

E. P. L. De Santis

Alexandre de Souza

Albert De Vries

Nicola Decaro

Chad Dechow

Hilton Deeth

Laure Deguillaume

Mel DeJarnette

Jack Dekkers

Maria del Pilar Schneider

Agnès Delacroix-Buchet

Manuel Delgado Pertiñez

Jeannine Delwiche

Chandraratne Dematawewa

Leo Dempfle

Michel Denis

Sylvie Desobry

Frédéric Destaillats

Estelle Devillard

Sarne DeVliegher

Trevor DeVries

Richard Dewhurst

Diego Di Genova

Jan Dijkstra

Nayil Dinkçi

Michael Diskin

Hilary Dobson

Joerg Dodenhoff

Marcus Doherr

Frigga Dohme

Shawn Donkin

Kristy Dorton

Zhengxia Dou

Larry Douglass

Maurice Doyon

James Drackley

MaryAnne Drake

Cord Drogemuller

Tom Druet

K.-J. Duan 
Jocelyn Dubuc

Luc Duchateau

Vincent Ducrocq

Eva Dudríková

Todd Duffield

Eliane Dumay

Susan Duncan

Susana Dunner

Robert Dyer

Alan Ealy

Maurice Eastridge

David Eckersall

Tom Edrington

James Ehrlich

Susan Eicher

Osman El Owni

Ibtisam E. M. El Zubeir

Daniel Elad

Gaber El-Baradei

Marina Elli

Kathryn Ellis

Steven Ellis

Theodore Elsasser

M. M. Eltholth

Francois Elvinger

Mauricio Elzo

Rodrigo Emediato

Marcia Endres

Francis Enjalbert

Saïd Ennahar

Lourens Erasmus

Danilo Ercolini

Richard Erdman

Georg Erhardt

Peter Erickson

Terry Etherton

Jong-Su Eun

Willard Eyestone

Michele Faccia

James Fadel

Alan Fahey

Thomas Famula

Nana Farkye

Harold Farrell

Vivek Fellner

James Ferguson

Jose Fernandez-Salguero

Isabel Ferreira

Alfred Ferret

Theodore Ferris

Sergei Fesenko

John Fetrow

Veerle Fievez

John Fike

Freddy Fikse

Ismail Filya

Raffaella Finocchiaro

Jeffrey Firkins
Richard FitzGerald

Monica Foote

Flavio Forabosco

Evelyn Forano

Andrew Forbes

Stephen Forsythe

Lawrence Fox

Bernadette Franco

Birgit Frank

Pina Fratamico

Gertraude Freyer

Paul Fricke

Theodore Friend

Nicolas Friggens

Pascal Froidevaux

George Fthenakis

Carmen Fuentes

Wendy Fulwider

John Fuquay

Matthew Gabler

Valérie Gagnaire

Andrzej Gamian

Rahul Reddy Gangidi

Ian Gardner

Randi Therese Garmo

Phil Garnsworthy

Dorian Garrick

Ana Garrido

Deborah Garruti

Frank Garry

Isabelle Gaucher

John Gay

Edvardas Gedgaudas

Nicolas Gengler

L. I. A. Gershenson

Katia Gianni

John Gibbons

Isabel Gigli

Rob Gilbert

Christiane Girard

Elizabeth Glass

Frederic Glasser

Sandra Godden

Lisbeth Goddik

Kevin Godinho

Ann Godkin

John Goeser

Arthur Goetsch

H. Douglas Goff

Jesse Goff

Luis Gomez Raya

Michael Gonda

Luciano González

Eliel González-Garcia

Oscar Gonzalez-Recio

Carlos Gonzalo

Ronald Gorewit

Shela Gorinstein
Kiev Gracias

Chris Grainger

Richard Grant

Carola Grebitus

Alice Green

Laura Green

Martin Green

Pablo Gregorini

Tanya Gressley

Lanny Griffin

Kenneth Griswold

Ab Groen

Yrjo Grohn

Ric Grummer

Charles Guard

Cristina Guedes

Manuel Guerra

Elisabetta Guerzoni

François Guillaume

Tim Guinee

Michael Gunderson

Benheng Guo

Mingruo Guo

Yan Guo

Abhijit Gurjar

Robert Gustafson

Beatriz Gutierrez-Gil

Frank Gwazdauskas

Jong Kyu Ha

Nam Joo Ha

Darryl Hadsell

George Haenlein

Kristin Hagen

Ilan Halachmi

Mary Beth Hall

Elin Hallén

Stacey Hamilton

Hedi Hammami

Harald Hammon

Lujia Han

Kathy Hanford

Mark Hanigan

Leslie Hansen

Peter J. Hansen

David Harmon

W. Harper

Bevin L. Harris

Joe Harrison

Steve Hart

Federico Harte

Gary Hartnell

Kevin Harvatine

Marie Haskell

John Hasler

Ashraf Hassan

Ronald Hatfield

Ali Hayaloglu

Ben Hayes 
Armagan Hayirli

J. Heesterbeek

Anna-Maija Heikkilä

Arlyn Heinrichs

Bill Herbert

Bjorg Heringstad

Anders Herlin

Horacio Hernandez

Marta Hernandez-Jover

Humberto Hernandez-Sanchez

Mette Herskin

Kasper Hettinga

Cord Heuer

Wolfgang Heuwieser

Clair Hicks

Peter Hildebrand

Arthur Hill

T. Hill

Eric Hillerton

Loic Hilliou

Bradley Hilty

J. Hinrichs

Christine Hinterhofer

Arnold Hippen

Gaby Hirsbrunner

Christina Hoelzel

Pat Hoffman

Joseph Hogan

Hendrik Hogeveen

Claus Holm

Mia Holmberg

Carl Holt

Kjell Holtenius

Menno Holzhauer

Brinton Hopkins

Jonathan Hopkinson

Russell Hovey

Mari Hovinen

Wayne Howard

Alex Hristov

John Hudson

Daniela Huessy

Pekka Huhtanen

Gerald Huntington

Walter Hurley

Catherine Hurtaud

Michael Hutjens

Juliana Huzzey

Erica Ruth Hynes

Stefania Iametti

Domenico Iannelli

Eveline Ibeagha-Awemu

Salam Ibrahim

Klaus Ingvartsen

Ignacio Ipharraguerre

Ruzaina Ishak

Galin Ivanov

Adam Jackanicz
Michel Jaffrin

Jette Jakobsen

Robert James

Janusz Jamrozik

Oliver Jann

Lee Ann Jaykus

Thomas Jenkins

Bruce Jenny

Margit Bak Jensen

Soren Jensen

Honglin Jiang

Zhihua Jiang

Rafael Jimenez-Flores

Eric Johansen

Kjell Johansson

Dave Johnson

Kristen Johnson

Mark Johnson

David Jones

Jamie Jonker

Ellen Jordan

Hossein Jorjani

Nitin Joshi

Sergio Juchem

Jarmo Juga

Vincent Juillard

S. Julia

Hans-Joachim Jung

Harry Kaiser

Sivaraj Kaliappan

Kenneth Kalscheur

Bernhard Kaltenboeck

Hachiro Kamada

D. H. Kang

Binnur Kaptan

Yonca Karagul Yuceer

Fatma Karakas Oguz

Elizabeth Karcher

Sanjay Karnati

Niel Karrow

R. Kasimanickam

Martin Kaske

Kerry Kaylegian

Gregory Keefe

Marcus Kehrli

Alan Kelly

Philip Kelly

David Kelton

J. Kennelly

David Kenny

Ronald Kensinger

Sandra Kentish

Jeffrey Keown

David Kerr

Alois Kertz

Hany Khalil

Ramesh Khanal

Hasan Khatib
Mehar Khatkar

A. Kilara

Jens Kildsgaard

Ronald Kincaid

Paul Kindstedt

Mark Kinsel

Haruki Kitazawa

Soressa Kitessa

Bengt Klarin

Daryl Kleinschmit

Joe Klemaszewski

Terry Klopfenstein

Joanne Knapp

Katharine Knowlton

Nurcan Koca

C. Kocharunchitt

Richard Kohn

Eric Kolver

Seyrani Koncagul

Sven König

Paul Kononoff

Hassan Korbekandi

Figen Korel

Sofya Korosteleva

Mikko Koskinen

Athanasios Koutinas

Asano Kozo

P. Krawczel

Prisca Kremer

Michael Kreuzer

Niels Kristensen

Michaela Kristula

Christa Kühn

Pradyuman Kumar

Limin Kung

Hae-Soo Kwak

Ilias Kyriazakis

Pierre Lacasse

Nicola Lacetera

Jeff Lakritz

Graham Lamb

Amy Lammert

Christoph Lammler

Thomas Lawlor

Andrew Laws

Yannick Le Cozler

Katharine Leach

Ian Lean

Stephen LeBlanc

Aat Ledeboer

Jung Lee

Sanghong Lee

Soo Lee

Alan Lefcourt

Andrés Legarra

Angelika Lehner

Claudia Leonardi

Jo Leroy 
Ken Leslie

Quanyang Li

M. Lidauer

Lena Lidfors

Silvia Liefers

Jamie Liegh

Ching Lin

Ole Lind

Raphael Linker

James Linn

Min-Tze Liong

John Lippolis

Noah Litherland

Xiao-Ming Liu

Zengting Liu

Anne Loberg

G. E. Lobley

Willie Loeffen

Scott Loeffler

Steven Loerch

Juan Loor

Ksenija Lopandic

Christelle Lopez

Leocadio Lopez

Evangelina Lopez de Maturana

Inmaculada López-Aliaga

Mario Lopez-Benavides

Rosina Lopez-Fandiño

Marta Lourenco

Peter Lovendahl

John Lucey

Matthew Lucy

Paul Ludden

Pilar Luna

Vicki Luna

Mogens Lund

Anne Lundén

Tim Lysyk

Sameer Mabjeesh

Nicolo Macciotta

Keith Macmillan

Ashkan Madadlou

Per Madsen

Elizabeth Maga

Johann Maierl

Harinder Makkar

Aduli Enoch Malau-Aduli

Bonnie Mallard

Christian Maltecca

Ephraim Maltz

Surajit Mandal

Michael Mangino

R. S. Manik

Esa Antero Mantysaari

C. Marce

Sophie Marchand

Jean Margerison

Primo Mariani
Pierre-Guy Marnet

Milan Marounek

Greg Martin

Thomas Martin

Sandra Martin del Campo

Cristina Martinez-Villaluenga

Silvana Martini

Roberto Matamoros

L. Mateus

Baltasar Mayo

Sinéad Mc Parland

Alan McAllister

Jack McAllister

Tim McAllister

Susan McCoard

David McCoy

Scott McDougall

Lee McDowell

Thomas McFadden

Mike McGilliard

Sean McGinn

Michael McGrath

Mark McGuire

Sheila McGuirk

Shawn McKenna

John McKillip

Carol McLaughlin

Kyle McLeod

Donald McMahon

John P. McNamara

Paul McSweeney

Margarita Medina

John Mee

Bhavbhuti Mehta

Susanne Meier

Ana Meikle

Graeme Mein

David J. Mellor

Erdogan Memili

Karensa Menzies

Uzi Merin

Juliana Messana

Serge Messier

Jos Metz

Lloyd Metzger

Theo Meuwissen

Heinrich Meyer

Matthew Meyer

Mireille Meylan

Marie-Caroline Michalski

Filippo Miglior

Yves Millemann

Craig Miller

Daniel Miller

Michael Miller

Robert Miller

Suzanne Millman

Despoina Miltiadou
Yoshinori Mine

Stephanie Minery

Giulietta Minozzi

Joshua Miron

Tom Misselbrook

Ignacy Misztal

Frank Mitloehner

Akio Miyamoto

Uzi Moallem

Peter Moate

Erwin Mollenhorst

Hugo Montaldo

Tera Montgomery

Jon Moorby

Dale Moore

Ofelia Mora

James Moran

Carmen Moraru

Pierre Morin

Paolo Moroni

Amir Mortazavian

Grith Mortensen

Ann Mothershaw

Kasey Moyes

F. Mozzi

Raphael Mrode

Richard Muck

C. Anne Muckle

Christoph Muelling

Herman Mulder

Isis Mullarky

Larry Muller

Michael Murphy

Richard Murray

T. Nagaraja

Tadashi Nakamura

Daryl Nash

Raymond Nebel

João Negrão

F. Neijenhuis

Brandon Nelson

Tamilee Nennich

Timothee Neuenschwander

C. Jamie Newbold

David Newburg

Eric Nicholson

Stephen Nickerson

Hanne Nielsen

Liza Nielsen

Soeren Nielsen

Akbar Nikkhah

Brian Nonnecke

Kenneth Nordlund

Richard Norell

H. Norman

David Notter

Hubert Nowakowski

Anna Nudda 
Karl Nuss

Daryl Nydam

Michael O'Connor

Michael O'Donovan

Keelin O'Driscoll

Jim O'Fallon

Luke O'Grady

Sean O'Keefe

Daniel O'Sullivan

Masahito Oba

Theresa Ochoa

Jørgen Ødegård

Nicholas Odongo

Garrett Oetzel

Shin Oikawa

Ammar Olabi

Richard Olde Riekerink

Carlos Oliveira

Stephen Oliver

Victor Olori

Katrina Olson

Pascal Oltenacu

James Oltjen

Nicole Olynk

Maria Oneca

Charles Onwulata

Bonney Oommen

Theo Oosthuizen

Geert Opsomer

Michael Orford

Henrik Østdal

Olav Osteras

Soren Ostergaard

Gernot Osthoff

Troy Ott

Joseph Ottobre

Michael Overton

Thomas Overton

William Owens

Barbaros Ozer

Max Paape

Colin Palmer

Donald Palmquist

Valentina Palucci

Lothar Panicke

Chung Park

Young Park

Peter Parodi

Matti Pastell

Francesca Patrignani

Ronald Pearson

Wilbert Pellikaan

P. Pelosi

M. Ángeles Pérez-Cabal

Miguel Perez-Enciso

Dolores Perez-Marin

Vincent Perreten

George Perry
Karin Persson Waller

Karl-Johan Petersson

Lennart Petersson

Christina Petersson-Wolfe

Hélène Petit

Kiro Petrovski

Wolfram Petzl

Adel Pezeshki

Arun Phatak

Clive Phillips

William Phillips

Gina Pighetti

Anne Pihlanto

Fabio Pilla

Jose Pires

Jan Plaizier

Yves Plante

Mélanie Plourde

Mario Poli

Geoffrey Pollott

Kayla Polzin

Ricardo Pong-Wong

Yves Pouliot

Maria Angeles Pozo Bayon

Jashbhai Prajapati

Aldo Prandini

Jennie Pryce

Young-Hee Pyo

Satu Pyorala

Phoebe Qi

Xuejun Qiu

Nicoletta Quaglia

Muhammad Subhan Qureshi

D. Owen Rae

Eran Raizman

Päivi Rajala-Schultz

Merceds Ramos

Ronald Randel

Maria Jose Ranilla

Scott Rankin

Morten Rasmussen

Robin Rastani

Kent Refsal

Shakeel Rehman

Douglas Reinemann

Martin Reist

Boulbaba Rekik

Bernard Remond

Jeffrey Reneau

Stefan Rensing

João Resende Júnior

Tony Reverter

Christopher Reynolds

Pedram Rezamand

Michelle Rhoads

Robert Rhoads

Ronald Richter

Manuela Rinaldi
Gonzalo Rincon

Robert Ringseis

Carlos Risco

Ali Risvanli

Allan Robins

John Roche

Lyle Rode

Jack Rodenburg

Luis Rodriguez

Maria Rodriguez-Prado

Dennis Romero

Gema Romero

Guilherme Rosa

Sandra Rose

Moshe Rosenberg

Marcelo Rosmini

C. Rotz

Maristela Rovai

Denis Roy

Melissa Royal

Pamela Ruegg

Duncan Runciman

Rachel Rupp

Jeffrey Rushen

Kenneth Rutherford

Zaeyoung Ryoo

R. G. Saacke

Hassan Sadri

Sina Safayi

A. Sahoo

Janeen Salak-Johnson

Ahmed Salama

Antonia Samoré

Juan Sanchez

Ashley Sanders

Carlos Sandoval-Castro

Carmen SanJose

Anderson Sant'Ana

Eduardo Santellano-Estrada

Jose Eduardo Santos

Marcos Santos

María Jesús Santos-Delgado

Mehdi Sargolzaei

James Sartin

Helga Sauerwein

Ioannis Savvaidis

Ashish Sawant

Brian Sayre

Larry Schaeffer

Walter Schaeren

Flavio Schenkel

Anke Schennink

Siegfried Scherer

Wes Schilling

David Schingoethe

Paul Schlosser

Amy Schmidt

Tonya Schoenfuss 
Friedrich Schöne

Jan Schonewille

Jon Schoonmaker

F. Schrick

Guillermo Schroeder

Ynte Schukken

Karin Schütz

Charles Schwab

M. Francesca Scintu

Raffaele Sciorsci

Bruce Seal

Philip Sears

Jean-Louis Sébédio

Henri Seegers

George Seidel Jr.

Kris Sejrsen

David Sela

Evan Sergeant

Magdalena Serrano

Asheber Sewalem

Anthony Seykora

Nagendra Shah

Avi Shamay

Roger Shanks

Randy Shaver

Jan Shearer

Martin Sheldon

Kei-ichi Shimazaki

Kevin Shingfield

George Shook

N. Shpigel

Marcos Vinicius Silva

William Silvia

Liam Sinclair

Harjinder Singh

Kuljeet Singh

Gregory Siragusa

William Sischo

Andrew Skidmore

Geof Smith

Gilbert Smith

John Smith

Julia Smith

K. Smith

Kelly Smith

Kevin Smith

Matt Smith

Stephen Smith

Trevor Smith

Warren Snelling

Charlie Sniffen

Gary Snowder

Michael Socha

Åse Sogstad

Robert Soliva-Fortuny

Morris Soller

Kei Sonoyama

Tad Sonstegard
Lorraine Sordillo

Morten Sørensen

Elena Sorrentino

Hélène Soyeurt

James Spain

Marisanna Speroni

Leon Spicer

Donald Spiers

Vitaly Spitsberg

Diane Spurlock

Srinand Sreevatsan

Judith Stabel

Katarzyna Stachowicz

Charles Stallings

Kim Stanford

Edward Stanisiewski

Charles Staples

James Steele

Alessandra Stella

Kerst Stelwagen

Roger Stephan

Marshall Stern

Jeffrey Stevenson

Tracy Stirling

Martin Stokes

William Stone

W. Marianne Stoop

Normand St-Pierre

Tomasz Strabel

Erling Strandberg

Ismo Stranden

Joseph Sturino

Guosheng $\mathrm{Su}$

Jessica Suagee

Anand Subramanian

Peter Sullivan

Jan Sunde

Catarina Svensson

Hermann H. Swalve

William Swecker

Matt Sylte

A. M. Tahamsbi

Miika Tapio

Suvi Taponen

Uri Tasch

Loren Tauer

M. Tegoni

Assadollah Teimouri Yansari

Evgenij Telezhenko

Ross Tellam

Seran Temelli

Marta Terre

Beverly Teter

William Thatcher

David Thomas

McFadden Thomas

Milton Thomas

Paul Thompson
Peter Thomsen

Jennifer Thomson

K. L. Thong

Walter Threlfall

Linda Tikofsky

Leo Timms

Evan Titgemeyer

Ashwani Tiwari

Nils Toft

Bert Tolkamp

Peggy Tomasula

Dana Tomlinson

Phillip Tong

Peter Tozer

Josephine Trott

Antonio-José Trujillo

Shogo Tsuruta

Cassandra Tucker

Michael Tunick

J. W. Tyler

Anna-Maria Tyrisevä

Susanne Ulbrich

Dan Undersander

Praveen Upreti

Martin Upsdell

Jorge Urioste

Zey Ustunol

Mette Vaarst

G. C. Vaillant

Eva Valdivia

Alessio Valentini

Georgios Valergakis

Arnould Valerie

Roger Vallejo

Michael Van Amburgh

Sarel van Amstel

Rik van der Tol

H. van Dorland

Frank van Eerdenburg

Diane Van Hekken

Jan-Thijs van Kaam

Jo Ann Van Kessel

Ariette van Knegsel

Jaap van Milgen

Lloyd Van Vleck

Michael VandeHaar

John VanLeeuwen

Paul VanRaden

Gabriella Varga

Mercedes Vazquez-Anon

Jeffrey Veenhuizen

Roel Veerkamp

Humberto Vega-Mercado

Roxana Verdini

Chris Verschoor

Indrakumar Vetharaniam

John Vicini

Johanna Vilkki 
Mar Villamiel

Beatriz Villanueva

Walkiria Viotto

E. Vlkova

Marina von Keyserlingk

Atte von Wright

Jean-Christophe Vuillemard

Natascha Vukasinovic

Adrianus Vuuren

Kevin Wade

Sarah Wagner

Susanne Waiblinger

Dan Waldner

Matthew Waldron

Eileen Wall

John Wallace

Pieter Walstra

Scott Waltner

Jiufeng Wang

Yuxi Wang

Paula Marie Ward

Lorin Warnick

Steven Washburn

D. C. Wathes

Michel Wattiaux

William Wavrin

Daniel Weary

Kim Weaver

Miriam Weber Nielsen

John Webster

Beat Wechsler

Neil Wedlock
Mingken Wei

Kent Weigel

Paul Weimer

Zwi Weinberg

Martin Weisbjerg

William Weiss

Joel Weller

Olga Wellnitz

William Wendorff

John Wenz

Randall Weselake

J. West

Tom Wheeler

I. M. S. White

Robert Whitlock

Lon Whitlow

George Wiggans

Lars Wiking

Andrea Wilcks

John Wilkinson

Cathleen Williams

David Wilson

Matthew Wilson

James Wilton

Christoph Winckler

Jack Windig

Wilhelm Windisch

Thomas Wittum

James Wohlt

Eckhard Wolf

David Wolfenson

Marie Wolfová
Millie Worku

Tom Wright

$\mathrm{Fu} \mathrm{Wu}$

Longfei $\mathrm{Wu}$

Qingping $\mathrm{Wu}$

Z. $\mathrm{Wu}$

Bob Xiang

Hariom Yadev

Wen Yang

Jesus Yaniz

Kyuichi Yasui

Fehmi Yazici

Yingwang Ye

Jessca Yee

Atilla Yetişmeyen

Zelalem Yilma

Allen Young

Fiona Young

Ruth Zadoks

Ricardo Zanella

Geoffrey Zanton

Alfonso Zecconi

Steve Zeng

Holm Zerbe

Feng-Qi Zhao

Xin Zhao

$\mathrm{Xu}$ Zhou

C. J. Ziemer

Richard Zinn

Bogdan Zisu

Birgit Zumbach 